Ann. Zootech., I977, 26 (I), I25-I 29.

Note

\title{
Relations entre la conformation et la composition des carcasses de bovins
}

\author{
B. L. DUMONT \\ Laboratoire de Recherches sur la viande \\ Centre national de Recherches zootechniques, I.N.R.A., \\ $7835^{\circ}$ Jouy-en-Josas (France)
}

\begin{abstract}
Résumé
On a étudié les relations existant, dans un échantilion commercial de bovins, entre la conformation jugée d'après la méthode normalisée de la Fédération Européenne de Zootechnie et la composition des carcasses appréciée par le rapport de la masse đésossée (C) (ensemble des muscles et des graisses) à la masse du squelette (S). Ce rapport $\mathrm{C} / \mathrm{S}$ augmente avec la note de conformation et avec le poids de carcasse. Le poids de carcasse et le rapport C/S expliquent ensemble 73 p. 100 de la variation de la note de conformation. Une amélioration de l'estimation de la masse désossée à partir de la note de conformation, à un poids et pour un niveau de conformation donnés, serait envisageable en complétant la méthode de jugement normalisé de la conformation par l'adjonction de critères liés à l'importance de l'ossature.
\end{abstract}

Si l'importance économique de la conformation des carcasses bovines est bien démontrée par son influence dans la détermination du prix du $\mathrm{kg}$ de carcasse (Dumont, ARnoux, I968) sa signification est encore controversée en raison de la faible variation biologique enregistrée, entre animaux, dans la distribution de la musculature (Dumont, Le Guelte et Arnoux i96i; Butterfield, r963). Pendant longtemps il a été délicat de préciser les conséquences technologiques de la variation de la conformation par suite du manque de méthodes de jugement global de ce caractère. On dispose maintenant, avec les propositions de de BoER et al. (1974), d'une méthode standardisée de description quantitative des carcasses de bovins qui autorise, sur la conformation et l'état d'engraissement, un classement précis et détaillé des bovins de différents types. On peut, dès lors, envisager d'analyser, par rapport à des références de conformation normalisées, quelles sont les consé- 
quences des variations enregistrées dans la pratique courante entre animaux de diverses classes commerciales.

La note présente les résultats obtenus dans une première comparaison de la note de conformation normalisée et de la composition de carcasses bovines.

On a considéré 40 gros bovins de différentes races comprenant des femelles $(\mathrm{N}=\mathrm{II})$, des mâles castrés $(\mathrm{N}=8)$ et des mâles entiers $(\mathrm{N}=2 \mathrm{I})$. Les animaux ont été choisis de manière à offrir un large échantillon de la variation constatée, pour la conformation, parmi les bovins de boucherie livrés sur le marché français. A ce titre l'analyse les concernant sera conduite sans tenir compte des différences d'origine sexuelle ou raciale existant entre eux.

Les animaux ont été sacrifiés dans les abattoirs du C.N.R.Z. Dès la fin des opérations d'abattage et de préparation des carcasses on a relevé le poids de la carcasse chaude $(\mathrm{P})$ en $\mathrm{kg}$. Vingt-quatre heures après, la carcasse était mesurée et jugée pour la conformation d'après les propositions faites par de BOER et al. (1974). La conformation des carcasses était appréciée par comparaison aux standards de référence des normes de charnure de la Fédération Européenne de Zootechnie selon la méthode proposée par Dumont, LE Guelte et Sornay (I975). Le jugement de la carcasse se faisait par pointage régional détaillé et donnait lieu à l'attribution d'une note pouvant varier entre 2 et $\mathbf{r} 6$.

Après une semaine de conservation en chambre froide à $+4^{\circ} \mathrm{C}$ on a pratiqué une coupe commerciale de la demi-carcasse droite et procédé au désossage des morceaux. Le total des masses osseuses enregistrées est considéré comme représentant la masse du squelette (S) de la demi-carcasse. On a enregistré, d'autre part, la masse des morceaux obtenus après coupe et désossage. La somme des masses des morceaux désossés (C) est considérée ici comme étant la masse des tissus recouvrant le squelette. Cette masse est essentiellement composée par l'ensemble des muscles et des graisses de la carcasse. La valeur du rapport C/S est retenue comme indice de la composition de la carcasse avant la coupe, au moment du jugement de sa charnure.

Le tableau I indique les valeurs de la moyenne, de l'écart-type et du coefficient de variation des caractères étudiés. D'après les normes de jugement de la F.E.Z. (Dumont, Le Guelte et Sornay, I975), la valeur moyenne correspond à des animaux de qualité bouchère satisfaisante aux profils musculaires rectilignes. Le poids de carcasse est cependant très supérieur à la moyenne nationale. Le tableau 2 indique la valeur des coefficients de corrélation entre les caractères de la carcasse.

Le rapport $\mathrm{C} / \mathrm{S}$ est lié de façon positive à la note de conformation. Au sein de la population étudiée il y a donc une liaison assez étroite entre le développement pondéral relatif des tissus par rapport au squelette et la conformation. Le modelé des formes des carcasses que traduit la note de conformation reflète donc une part importante de la variation de leur composition anatomique tissulaire, au plan de l'équilibre existant entre le squelette et la masse des tissus qu'il supporte. Cette observation va dans le même sens que les résultats de BERGström (I974) qui a constaté des variations dans le rapport de charnure des carcasses des différentes classes néerlandaises de qualité. Ces résultats sont concordants aussi avec ceux de Kauffman, Smith et Long (I970) qui ont établi, dans la composition relative de lots de carcasses de conformation différente (types " ectomorphe " et " méso- 
TABLEAU I

Valeur des caractères de la carcasse

Carcass characteristics

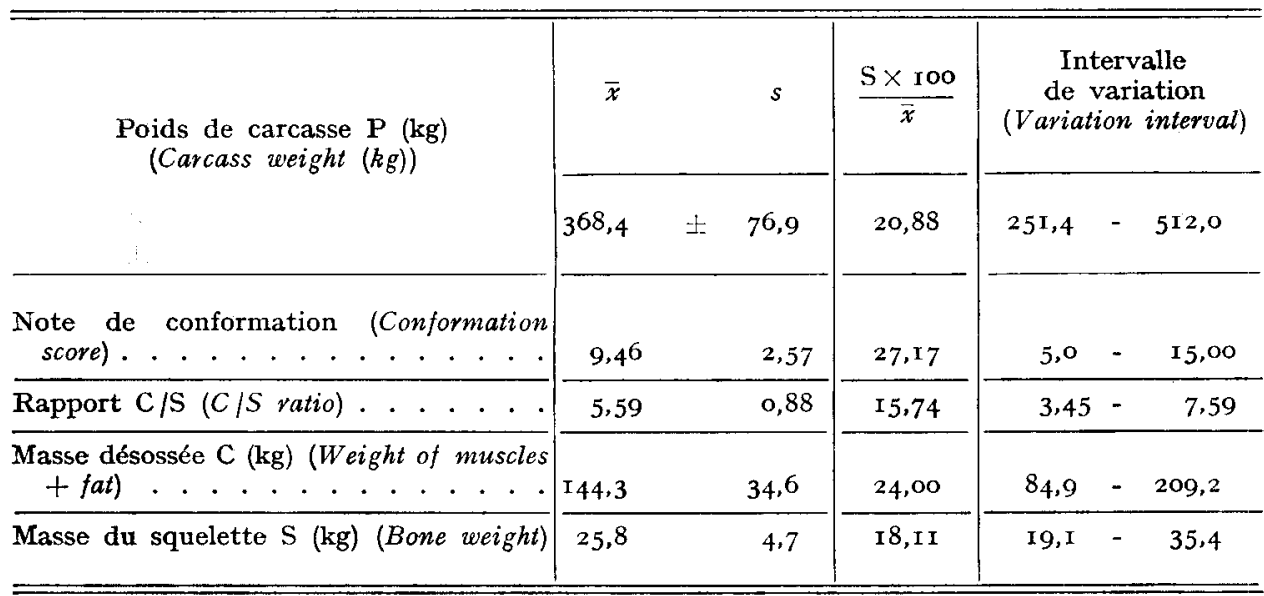

TABLEAU 2

Relations entre les caractères de la carcasse

Relationships between cancass characteristics

\begin{tabular}{|c|c|c|c|c|}
\hline & $\mathbf{P}$ & $\mathrm{C}$ & $\mathrm{C} / \mathrm{S}$ & $\mathbf{N}$ \\
\hline Poids de la carcasse $\mathbf{P}$ (Carcass weight) . . . . . & $1 / 1$ & 0,99 & 0,59 & $0,8 \mathrm{x}$ \\
\hline Masse désossée C (Muscles + fat) . . . . . . & & $1 / 1$ & 0,67 & 0,85 \\
\hline $\begin{array}{l}\text { Rapport de la masse désossée à la masse du sque- } \\
\text { lette (C/S) (Muscles + fat /bone ratio). . . . . }\end{array}$ & & & 111 & $0,7^{\circ}$ \\
\hline Note de conformation $\mathrm{N}$ (Conformation score). . . & & & & 111 \\
\hline
\end{tabular}

morphe ") des différences allant dans le sens d'une meilleure charnure en faveur des carcasses les mieux conformées.

Une part de la variation enregistrée, dans notre échantillon, dans la note de conformation et la composition est attribuable à la variation de poids des carcasses. Le poids est, en effet, lié à la fois à la note de conformation $(r=+0,8 \mathrm{I})$ et au rapport $\mathrm{C} / \mathrm{S}(r=+0,70)$. L'ensemble de la masse désossée $\mathrm{C}$ croît relativement plus rapidement que le poids de la carcasse, comme l'indique l'étude de la régression du logarithme de $\mathrm{C}$ en fonction du logarithme de $\mathrm{P}$ qui met en évidence une allométrie majorante de $\mathrm{C}$ dans l'ensemble de la carcasse :

$$
\log \mathrm{C}=\mathrm{I}, \mathrm{I} 66 \log \mathrm{P}-0,834 \quad(r=+0,99 \mathrm{I})
$$

Il est donc normal d'assister, avec l'augmentation de poids, à un accroissement du rapport $\mathrm{C} / \mathrm{S}$ des carcasses. En corollaire on doit s'attendre aussi à une augmen- 
tation de la note de conformation, si l'on pose que la conformation se définit par l'épaisseur des tissus par rapport aux dimensions du squelette qui les supporte (Dumont, Le Guelte et Sornay, 1975). Effectivement le poids de carcasse et le rapport C/S expliquent, à eux deux, 73 p. roo de la variation de la note de conformation.

A poids de carcasse constant, la corrélation partielle entre la note de conformation et le rapport $C / S$ est seulement de $+0,47$, ce qui indique qu'une part importante de la variation de ce caractère n'est pas reflétée par la note accordée à la conformation. Ceci permettrait de comprendre les faibles différences constatées parfois dans la pratique entre carcasses de conformation différentes, dans des contrôles de rendement commerciaux en viande désossée qui amènent leurs auteurs à conclure que la conformation ne donne guère d'information sur le rendement en viande réel de la carcasse (SORnay et BobIon, I974; Priou, Sornay et Soudan, I976).

La relation un peu plus étroite, établie, à poids de carcasse constant, entre la note de conformation et la masse désossée $C(r=+0,58)$ suggère que le jugement de la conformation, tel qu'il a été pratiqué, reflète mieux le développement absolu des tissus que leur importance relative par rapport au squelette. Ceci peut s'expliquer par le fait que la méthode des profils musculaires utilisée pour apprécier la conformation (Houdinière I957; Dumont, Le Guelte et Sornay, I975), s'intéresse au développement apparent de la musculature par rapport à des lignes théoriques passant par des points de repère fixes, sans tenir compte essentiellement de l'importance des supports osseux. On peut penser qu'on améliorerait la valeur prédictive de la composition de la carcasse à partir de la note de conformation, en considérant en plus du jugement des profils musculaires, des caractères directement liés à l'importance du squelette (par exemple les dimensions — diamètre transversal ou périmètre — du tarse au niveau de l'articulation tarso-métatarsienne).

Ces résultats amènent à penser d'autre part, que le jugement précis et détaillé de la conformation réalisé dans cette étude (appréciation des carcasses sous différents angles, par référence à des standards photographiques très nuancés) prend en compte des éléments plus complexes que ceux qui déterminent simplement l'importance en masse des tissus. On peut ainsi avancer que le jugement de la conformation intéresse les profils externes des carcasses dans certaines directions où le développement musculaire - par rapport à son support osseux - modèle de façon particulière le volume ou la forme des tissus sans en modifier sensiblement ni l'importance absolue, ni l'importance relative. Cette hypothèse mériterait d'être vérifiée par des études ultérieures où la conformation serait à la fois appréciée par la méthode de référence de la F.E.Z. et définie par des mesures objectives de volume ou de forme des diverses régions de la carcasse.

Rȩ̧u pour publication en octobre 1976.

\section{Remerciements}

Ce travail a été réalisé avec la collaboration de M. Roy et avec l'aide de M. Morand (de l’I.T.E.B.) que nous tenons à remercier pour leur coopération. 


\section{Summary}

\section{Relationships between conformation and composition of bovine carcasses}

Relationships between conformation and composition were studied on a sample of 40 carcasses of various commercial types. Conformation was scored according to the standardized method proposed by the European Association for Animal Production (Livestock Prod. Sci., I974 (I), r 5I-I64). Composition was estimated by the ratio: weight of muscles + fat/bone weight. This ratio increased with conformation score and carcass weight. Carcass weight and composition both accounted for $73 \%$ of the confcrmation score variation. The assessment of the amount of flesh in the carcass, at a given weight and confcrmation level, would be improved by introducing in the scoring method for carcass conformation some additional criteria related to bone development.

\section{Références bibliographiques}

Bergström P. L., I974. Slachtkwaliteit bij runderen. I43 pp. Centre for Agricultural Publishing and Documentation, Édit. WAGENINGEN.

De Boer H., Dumont B. L., Pomeroy R. W., Weniger J. H., i974. Manual on E.A.A.P. Reference methods for the assessment of carcass characteristics in cattle. Livestock Prod. Sci., 1, I $5 \mathrm{~T}-\mathrm{I} 64$.

Butterfield R. M., 1963. Relative growth of the musculature of the ox. in Symp. Carcase composition and Appraisal of Meat Animals-Paper 7-I C.S.I.R.O. Melbourne.

Dumont B. L., Arnoux J., I968. Influence de différents caractères de la carcasse de bovins sur la détermination de son prix. Études de Statistique agricole, 1, 1-30, Office statistique des communautés européennes.

Dumont B. L., Le Guelte P., Arnoux J., i961. Étude biométrique des bovins de boucherie I. Variabilité de la composition anatomique de la carcasse des bovins charollais. Ann. Zootech., 10, I 49-I 54 .

Dumont B. L., Le Guelte P., Sornay J., 1975. Le jugement de la conformation des carcasses de bovins. Technipel édit. Paris, $26 \mathrm{pp}$.

Houdinik̀re A., 1957. L'examen des "profils musculaires " dans l'appréciation de la qualité des viandes. Bull. Acad. Vét., 30, 5I-62.

Kauffman R. G., Smith R. E., Long R. A., I970. Bovine topography and its relationship to composition. Proc. $23 r d$ Ann. Rec. Meat Cont. A.M.S.A., Ioo-i I7.

Priou J., Sornay J., Soudan J. P., 1976. Conditions pratiques d'utilisation des carcasses de jeunes bovins dans un circuit commercial témoin. ITEB édit. Paris, 64 pp.

Sornay J., Bobion D., I974. Aptitude à l'emploi des carcasses de taurillons limousins. ITEB édit. Paris, 5 I pp. 